\title{
MAXIMUM AND MINIMUM FIRST EIGENVALUES FOR A CLASS OF ELLIPTIC OPERATORS
}

\section{CARLO PUCCI ${ }^{1}$}

Let $A$ be an open bounded set of $R^{m}, \alpha$ a positive constant, $\alpha \leqq(1 / m)$; let $\mathfrak{L}_{\alpha}$ be the class of elliptic differential operators

$$
L \equiv \sum_{i, j}^{1, m} a_{i j}(x) \frac{\partial^{2}}{\partial x_{i} \partial x_{j}},
$$

with $a_{i j}$ measurable in $A$ and satisfying in $A$ the conditions

$$
\sum_{i, j}^{1, m} a_{i j}(x) p_{i} p_{j} \geqq \alpha|p|^{2}, \quad \sum_{i=1}^{m} a_{i i}(x)=1 .
$$

Observe that $(1 / m) \Delta$ belongs to $\mathfrak{L}_{\alpha}$. Let $G$ be the class of functions $u$ such that $u$ is continuous in $\bar{A}, u \in H^{2, m}(A), u=0$ in $\partial A, u$ positive in $A$. Let $\Lambda$ be the set of real numbers $\lambda$ for which there is an $L$ in $\mathfrak{L}_{\alpha}$ and a function $u$ in $G$ such that

$$
L u+\lambda u=0 \quad \text { a.e. in } A .
$$

By the maximum principle all the $\lambda$ 's are positive (see [1]). Bounds for the $\lambda$ 's were established under various hypotheses by Duffin [2], Protter-Weinberg [3]. Here we want to determine if $\Lambda$ has a maximum or a minimum and for what operator in $\mathfrak{L}_{\alpha}$ the maximum or minimum occurs.

Let $M_{\alpha}, m_{\alpha}$ denote the maximizing and minimizing operator relative to the class $\mathfrak{L}_{\alpha}$, that is for each fixed function $u$ and fixed $x$ :

$$
M_{\alpha}[u(x)]=\sup _{L \in \mathfrak{L}_{\alpha}} L u(x), \quad m_{\alpha}[u(x)]=\inf _{L \in \mathfrak{L}_{\alpha}} L u(x),
$$

with $u$ in $G$. (For definitions and general properties of such operators see [5] and, in the case of two variables, see also [4].)

Theorem I. Let $\partial A$ be of class $C^{2}$. If there is a function $u_{1}\left(u_{2}\right)$ of class $G$ and a constant $\lambda^{\prime}\left(\lambda^{\prime \prime}\right)$ such that

$$
\begin{aligned}
M_{\alpha}\left[u_{1}\right]+\lambda^{\prime} u_{1}=0 & \text { a.e.in } A, \\
m_{\alpha}\left[u_{2}\right]+\lambda^{\prime \prime} u_{2}=0 & \text { a.e. in } A,
\end{aligned}
$$

then $\Lambda$ has a minimum (a maximum) and

Received by the editors February 9, 1966.

1 This research was done in part at Louisiana State University. The author thanks Dr. A. Zettl for helpful discussions. 


$$
\lambda^{\prime}=\min \Lambda, \quad \lambda^{\prime \prime}=\max \Lambda .
$$

THEOREM II. Let $A$ be the sphere $\{x:|x|<1\}$. Denote with $b(c)$ the first zero of the Bessel function $J_{p}\left(J_{q}\right)$ where

$$
p=\frac{2(m-1) \alpha-1}{2-2(m-1) \alpha}, \quad q=\frac{1-2 \alpha}{2 \alpha} .
$$

Then

$$
\min \Lambda=[1-(m-1) \alpha] b^{2}, \quad \max \Lambda=\alpha c^{2} ;
$$

the equation for which the minimum occurs is

$$
\sum_{i, j}^{1, m}\left[\delta_{i j}+(1-m \alpha) \frac{x_{i} x_{j}}{|x|^{2}}\right] \frac{\partial^{2} u}{\partial x_{j} \partial x_{j}}+[1-(m-1) \alpha] b^{2} u=0
$$

and a solution of class $G$ is

$$
|x|^{-p} J_{p}(b|x|)
$$

the equation for which the maximum occurs is

$$
\sum_{i, j}^{1, m}\left[\frac{1-\alpha}{m-1} \delta_{i j}+\frac{m \alpha-1}{m-1} \frac{x_{i} x_{j}}{|x|^{2}}\right] \frac{\partial^{2} u}{\partial x_{i} \partial x_{j}}+\alpha c^{2} u=0,
$$

and a solution of class $G$ is

$$
|x|-q J_{q}(c|x|) \text {. }
$$

Observe that for $\alpha=1 / m$ there is only the operator $(1 / m) \Delta$ in the class $\mathscr{L}_{\alpha}$ and by Theorem II it follows that $\min \Lambda=\max \Lambda=m / b$ with $b$ the first eigenvalue of $J_{(m-2) / 2}$.

THEOREM III. Let $r_{1}$ be the supremum of the radii of the spheres contained in $A$ and $r_{2}$ the infimum of the radii of the spheres containing $A$. Then for any $\lambda$ in $\Lambda$ we have

$$
\frac{1-(m-1) \alpha}{r_{2}^{2}} b^{2} \leqq \lambda \leqq \frac{\alpha}{r_{1}^{2}} c^{2},
$$

where $b, c$ are defined in Theorem II.

First we prove a lemma:

Lemma. Let $\partial A$ be of class $C^{2}$ and let $u$ in $G$ be a solution of (3) for some $L$ in $\mathfrak{L}_{\alpha}$ and some real $\lambda$. Denote by $d(x)$ the distance of $x$ from $\partial A$; there are two positive constants $k_{1}, k_{2}$ such that in $A$

$$
k_{1} d(x) \leqq u(x) \leqq k_{2} d(x)
$$


The lemma is substantially known; the proof is based on the standard use of an auxiliary function.

By hypothesis there is a constant $h$ such that for any point $x^{o}$ of $\partial A$ two open spheres $S_{1}$ and $S_{2}$ of radius $h$ exist such that $S_{1}$ is contained in $A, S_{2}$ has no points in common with $A$ and $x^{\circ}$ belongs to the closure of both $S_{1}$ and $S_{2}$. For a fixed $x^{o}$ of $\partial A$ let $y$ be the center of $S_{2}$ and

$$
g(y)=1-\left(\frac{h}{|x-y|}\right)^{1 / \alpha} .
$$

From (1) it follows that

$$
\begin{aligned}
L g= & \frac{1}{2}\left(\frac{h}{|x-y|}\right)^{1 / \alpha}|x-y|^{-2} \\
& \cdot\left\{1-\left(2+\frac{1}{\alpha}\right) \sum_{i, j}^{1, m} a_{i j} \frac{\left(x_{i}-y_{i}\right)\left(x_{j}-y_{j}\right)}{|x-y|^{2}}\right\}
\end{aligned}
$$

and from (2)

$$
L g<-c \text { in } A \text {, }
$$

with $c$ a positive constant depending only on $\alpha, h$ and the diameter of $A$. Denote by $t$ the maximum of $\lambda u$ in $\bar{A}$; we obtain

$$
L\left(\frac{t}{c} g-u\right) \leqq 0 \text { in } A, \quad \frac{t}{c} g-u \geqq 0 \text { in } \partial A,
$$

and by the maximum principle (see [1])

$$
u \leqq \frac{t}{c}\left[1-\left(\frac{h}{|x-y|}\right)^{1 / \alpha}\right] .
$$

For a fixed $x$ in $A$ denote by $x^{o}$ one of the nearest points to $x$ on $\partial A$; we have $\left|x-x^{\circ}\right|=d(x),|x-y|=d(x)+h$ and by (17) the second inequality of (14) follows for a proper choice of $k_{2}$.

Now let $y$ be the center of the sphere $S_{1}$ relative to a point $x^{\circ}$ in $\partial A$, and let

$$
\begin{aligned}
T & \equiv\left(x: \frac{h}{2}<|x-y|<h\right), \\
s & =\frac{h}{2^{1 / \alpha}-1} \min _{a(x) \geqq h / 2} u(x)
\end{aligned}
$$

it follows that 


$$
u+s g \geqq 0 \text { in } \partial T
$$

and by (16)

$$
L(u+s g)=-\lambda u+s L g<0 \text { in } T,
$$

then again using the maximum principle

$$
u(x) \geqq s\left\{\left(\frac{h}{|x-y|}\right)^{1 / \alpha}-1\right\} \text { in } T .
$$

For a fixed $x$ in $A$, with $d(x)<(h / 2)$, denote by $x^{o}$ the nearest point of $\partial A$; then $|x-y|=h-d(x)$ and the first inequality of (14) follows by (18); for $x$ such that $d(x) \geqq(h / 2)$ it follows by the positivity of $u$.

Proof of Theorem I. Suppose $u_{1}$ in $G$ is a solution of (5).

By a previous result (see [3]) there is an operator $L_{1}$ in $\mathscr{L}_{\alpha}$ such that

$$
M_{\alpha}\left[u_{1}\right]=L_{1} u_{1} \text {. }
$$

Let $u$ be a function of $G$ which is a solution of (3), with $L$ in $\mathscr{L}_{\alpha}$. By the lemma

$$
\inf _{x \in A} \frac{u_{1}(x)}{u(x)}=t>0
$$

We have

$$
\lambda s u-\lambda^{\prime} u_{1} \leqq 0 \text { in } A \text { for } s=t \frac{\lambda^{\prime}}{\lambda}
$$

by (4) we obtain

$$
L\left(s u-u_{1}\right) \geqq L s u-M_{\alpha}\left[u_{1}\right]=-\lambda s u+\lambda^{\prime} u_{1} \geqq 0 \text { a.e. in } A .
$$

Also $s u-u_{1}=0$ in $\partial A$ and by the maximum principle $s u-u_{1} \leqq 0$ in $A$, that is

$$
\frac{\lambda^{\prime}}{\lambda} \leqq \frac{1}{t} \frac{u_{1}}{u} \text { in } A
$$

and $\lambda^{\prime} \leqq \lambda$ follows by definition of $t$.

A similar proof holds for the maximum of $\Lambda$; in this case we consider

$$
\sup _{x \in A} \frac{u_{2}(x)}{u(x)},
$$

which is finite by the lemma.

Proof of Theorem II. By (8) and (2) it follows that $p$ and $q>-1$. 
Let $r$ be the distance from the origin and $\phi(r)$ the function given by (13). By elementary properties of Bessel functions

$$
\phi(r)=\sum_{i=0}^{\infty}(-1)^{i}\left(\frac{c}{2}\right)^{2 i+q} \frac{r^{2 i}}{i ! \Gamma(i+q+1)},
$$

and $\phi$ is nonnegative, decreasing and of class $C^{2}$ in $[0,1]$,

$$
\phi^{\prime}(0)=0, \quad \phi(1)=0 ;
$$

furthermore $\phi$ is a solution of the equation

$$
\phi^{\prime \prime}+\frac{1+2 q}{r} \phi^{\prime}+c^{2} \phi=0
$$

that is

$$
\alpha \phi^{\prime \prime}+\frac{1-\alpha}{r} \phi^{\prime}+c^{2} \alpha \phi=0
$$

We prove that

$$
\phi^{\prime \prime}-\frac{\phi^{\prime}}{r} \geqq 0 \text { in }(0,1]
$$

By (20)

$$
\phi^{\prime \prime}-\frac{\phi^{\prime}}{r}=-\frac{1}{\alpha} \frac{\phi^{\prime}}{r}-c^{2} \phi .
$$

Inequality (21) holds in the limit at $r=0$ and, by (22) it holds for $r=1$. If (21) does not hold

$$
-\frac{1}{\alpha} \frac{\phi^{\prime}}{r}-c^{2} \phi
$$

has a minimum in $(0,1)$ and its derivative is zero at this point, that is

$$
-\frac{1}{\alpha} \frac{\phi^{\prime \prime}}{r}+\frac{1}{\alpha} \frac{\phi^{\prime}}{r^{2}}-c^{2} \phi^{\prime}=0
$$

and by (20) it follows that at the point of minimum (23) is positive.

Let $u(x)=\phi(r) ; u$ belongs to $G$. Let $C_{i}[u]$ be the principal curvatures of $u$, i.e. the eigenvalues of the matrix $\left|\left(\partial^{2} u\right) /\left(\partial x_{i} \partial x_{j}\right)\right|$ ordered in the following way

$$
C_{1}[u] \leqq C_{2}[u] \leqq \cdots \leqq C_{m}[u]
$$


By a previous result, see [3],

$$
m_{\alpha}[u]=[1-(m-1) \alpha] C_{1}[u]+\alpha \sum_{i=2}^{m} C_{i}[u] .
$$

By (21)

$$
C_{m}[u]=\phi^{\prime \prime}, \quad C_{i}[u]=\frac{\phi^{\prime}}{r}, \quad i=1,2, \cdots, m-1,
$$

then by (20)

$$
m_{\alpha}[u]+c^{2} u=0 \text { in } A \text {. }
$$

The second equality of (9) follows by Theorem I. Since

$$
\frac{\partial^{2} u}{\partial x_{i} \partial x_{j}}=\phi^{\prime \prime} \frac{x_{i} x_{j}}{r^{2}}+\phi^{\prime}\left(\frac{\delta_{i j}}{r}-\frac{x_{i} x_{j}}{r^{3}}\right),
$$

(12) follows.

The function $\phi(r)$ defined by (11) is a solution of

$$
[1-(m-1) \alpha] \phi^{\prime \prime}+\frac{(m-1) \alpha}{r} \phi^{\prime}+[1-(m-1) \alpha] b^{2} \phi=0 .
$$

By the same argument (21) holds and hence also (24) with $u(x)=\phi(r)$. Similarly, using

$$
M_{\alpha}[u]=[1-(m-1) \alpha] C_{m}[u]+\alpha \sum_{i=1}^{m-1} C_{i}[u],
$$

we complete the proof.

Proof of TheOREM III. Let $r$ be the radius of a sphere $S$, with $\bar{A} \subset S$. By Theorem II there follows the existence of a function $v$, $v \in H^{2, m}(S) \cap C^{o}(\bar{S})$, such that

$$
\begin{aligned}
M_{\alpha} v+\frac{\alpha c^{2}}{r^{2}} v & =0, \quad v>0 \text { in } S, \\
v & =0 \text { in } \partial S .
\end{aligned}
$$

Let

$$
t=\max _{x \in \bar{A}} \frac{u(x)}{v(x)}
$$

where $u$ is a function of class $G$ and a solution of (3) with $L \in \mathscr{L}_{\alpha}$.

The nonpositive function $u-t v$ takes its maximum inside $A$ and at that point $L(u-t v) \leqq 0$; but 


$$
L(u-t v) \geqq-\lambda u+t \frac{\alpha c^{2}}{r^{2}} v=\lambda(t v-u)+t v\left(\frac{\alpha c^{2}}{r^{2}}-\lambda\right) \text { in } A .
$$

Hence

$$
\frac{\alpha c^{2}}{r^{2}}-\lambda \leqq 0
$$

Observation I. In Theorem I the hypothesis "Let $\partial A$ be of class $C^{2}$ " can be replaced by "Let $A$ be star-shaped." The hypothesis of smoothness of the boundary was used only to prove that

$$
\inf _{x \in A} \frac{u_{1}(x)}{u(x)}>0 .
$$

Using a dilatation we can replace $u_{1}$ by a function $v$ such that

$$
v>0 \text { in } \bar{A}, \quad M_{\alpha} v+\bar{\lambda} v=0 \text { in } A,
$$

with $\bar{\lambda}$ as near as we like to $\lambda^{\prime}$. By the argument of the proof of Theorem $I$ it follows that $\lambda \geqq \bar{\lambda}$ and then $\lambda \geqq \lambda^{\prime}$.

A similar argument holds for the maximum of $\Lambda$.

Observation II. In Theorem I the hypothesis "Let $\partial A$ be of class $C^{2}$ " can be replaced by an hypothesis used by Duffin in a similar problem [2]: "We suppose that a function $w$ of class $C^{2}(\bar{A})$ exists such that

$$
w>1 \text { in } A, \quad M_{\alpha} w+k w<0 \text { in } \partial A,
$$

where $k=\sup \Lambda . "$

We observe that if (25) holds the proof is the same as before. If (25) does not hold, for $\epsilon$ positive and sufficiently small

$$
\frac{u_{1}+\epsilon w}{u}
$$

takes its minimum in a point so near to the boundary that there $M_{\alpha} w+k w<0$. In this point of minimum we must have

$$
L \frac{u_{1}+\epsilon w}{u} \geqq 0,
$$

and by the hypothesis and some computations it follows that $\lambda^{\prime} \leqq \lambda$. The other part of the proof is obtained considering

$$
\frac{u_{2}}{u+\epsilon w} \text {. }
$$


Observation III. Let $\beta, \gamma$ denote two nonnegative constants; the previous considerations can be extended to the class $\mathscr{L}_{\alpha, \beta, \gamma}$ of elliptic operators $L$

$$
L=L_{1}+\sum_{i=1}^{m} b_{i} \frac{\partial}{\partial x_{i}}+c
$$

with $b_{i}, c$ measurable in $A$ and

$$
L_{1} \in £_{\alpha}, \quad \sum_{i=1}^{m} b_{i}^{2} \leqq \beta^{2}, \quad-\gamma \leqq c \leqq 0 .
$$

The maximizing and minimizing operator related to the class $\mathfrak{L}_{\alpha, \beta, \gamma}$ is studied in [5].

\section{REFERENCES}

1. A. D. Aleksandrov, Certain estimates for the Dirichlet problem, Dokl. Akad. Nauk SSSR 134 (1960), 1001-1004=Soviet Math. Dokl. 1 (1961), 1151-1154.

2. R. J. Duffin, Lower bounds for eigenvalues, Phys. Rev. 71 (1947), 827-828.

3. M. H. Protter and H. F. Weinberger, On the spectrum of general second order operators, Bull. Amer. Math. Soc. 72 (1966), 251-255.

4. C. Pucci, Un problema variazionale per $i$ coefficienti di equazioni differenziali di tipo ellittico, Ann. Scuola Norm. Sup. Pisa 16 (1962), 159-172.

5. - O Operatori ellittici estremanti, Ann. Mat. Pura Appl. 72 (1966), 141-170.

UNIVERSITA DI GENOVA 